\title{
SPIRAL WAVES TRIGGERED BY SHADOWS IN TRANSITION DISKS
}

\author{
Matías Montesinos $^{1,2}$, Sebastian Perez ${ }^{1,2}$, Simon Casassus $^{1,2}$, Sebastian Marino ${ }^{1,2}$, \\ Jorge CuAdra ${ }^{2,3}$, AND VAlentin Christiaens ${ }^{1,2}$ \\ ${ }^{1}$ Departamento de Astronomía, Universidad de Chile, Casilla 36-D, Santiago, Chile; montesinos@das.uchile.cl \\ ${ }^{2}$ Millennium Nucleus "Protoplanetary Disks," Chile \\ ${ }^{3}$ Instituto de Astrofísica, Pontificia Universidad Católica de Chile, Santiago, Chile \\ Received 2016 January 27; revised 2016 April 5; accepted 2016 April 16; published 2016 May 13
}

\begin{abstract}
Circumstellar asymmetries such as central warps have recently been shown to cast shadows on outer disks. We investigate the hydrodynamical consequences of such variable illumination on the outer regions of a transition disk, and the development of spiral arms. Using 2D simulations, we follow the evolution of a gaseous disk passively heated by the central star, under the periodic forcing of shadows with an opening angle of $\sim 28^{\circ}$. With a lower pressure under the shadows, each crossing results in a variable azimuthal acceleration, which in time develops into spiral density waves. Their pitch angles evolve from $\Pi \sim 15^{\circ}-22^{\circ}$ at the onset, to $\sim 11^{\circ}-14^{\circ}$, over $\sim 65 \mathrm{au}$ to $150 \mathrm{au}$. Self-gravity enhances the density contrast of the spiral waves, as also reported previously for spirals launched by planets. Our control simulations with unshadowed irradiation do not develop structures, except for a different form of spiral waves seen at later times only in the gravitationally unstable control case. Scattered light predictions in the $H$-band show that such illumination spirals should be observable. We suggest that spiral arms in the case-study transition disk HD 142527 could be explained as a result of shadowing from the tilted inner disk.
\end{abstract}

Key words: accretion, accretion disks - hydrodynamics - methods: numerical - planets and satellites: dynamical evolution and stability - protoplanetary disks

\section{INTRODUCTION}

During the last decade optical-infrared direct imaging of circumstellar disks has revealed spiral patterns around some HAeBe stars. These spirals are seen in intermediate mass stars, $\sim 2 M_{\odot}$, with an inner cavity in a gas-rich disk, and are loosely classified as transition disks (e.g., Espaillat et al. 2012). Outstanding examples are AB Aur (e.g., Grady et al. 1999; Fukagawa et al. 2004), HD 100546 (e.g., Grady et al. 2001; Boccaletti et al. 2013), HD 142527 (e.g., Fukagawa et al. 2006; Casassus et al. 2012a), MWC 758 (e.g., Grady et al. 2013; Benisty et al. 2015), HD 135344B (e.g., Muto et al. 2012a; Garufi et al. 2013; Wahhaj et al. 2015), and HD 100453 (Wagner et al. 2015). Spirals usually stem away from the outer rims of disk cavities, with large pitch angles $\left(10^{\circ}-15^{\circ}\right.$, Dong et al. 2015b). They can extend from $\sim 15$ au to 600 au from the central star (Clampin et al. 2003; Christiaens et al. 2014) and some of them show remarkable $m=2$ azimuthal symmetry (Dong et al. 2015b).

The origin of such spirals is motivating intense research efforts. Spiral density waves can be launched by unseen substellar companions of $\gtrsim M_{\text {jup }}$ (Muto et al. 2012b). Juhász et al. (2015) studied the observability of spirals launched by embedded planets, suggesting that they are the result of changes in the vertical scale height of the disk rather than density perturbations (see also Pohl et al. 2015). Interestingly, the large pitch angles in spirals with $m=2$ symmetry can be explained by the presence of massive planets exterior to the spiral features (Dong et al. 2015b). An origin in gravitational instabilities (GI) is also plausible for massive disks, but limits the size of the spirals to $\lesssim 100$ au (e.g., Lodato \& Rice 2004; Dong et al. 2015a). Thus, current spiral models require either massive planets, gravitationally unstable disks, or both (Pohl et al. 2015).
Motivated by the recent identification of deep shadows cast by an inner warp in HD 142527 (Casassus et al. 2015; Marino et al. 2015), in this Letter we consider the dynamical consequences of the temperature being forced onto the outer disk as it periodically flows under such shadows. We use twodimensional (2D) hydro simulations to follow the evolution of a passive gaseous disk subjected to non-axially symmetric shadowing, and report on the development of spiral waves (Section 2). Based on these results (Section 3), we propose an alternative mechanism to trigger spirals from illumination effects in the outer regions of gapped systems (Section 4).

\section{THE MODEL}

We are interested in the evolution of a self-gravitating, planetless, gaseous circumstellar passive disk. The stellar radiation field is fixed to two reference values, $L_{\star}=1 L_{\odot}$, and $L_{\star}=100 L_{\odot}$. We consider disks with masses $M_{\mathrm{d}}=0.05$, and $M_{\mathrm{d}}=0.25 M_{\star}$, without including viscous dissipation, which translates into very low accretion rates. A constant kinematic viscosity prescription is used, given by $\nu=4.5 \times 10^{7} \mathrm{~m}^{2} \mathrm{~s}^{-1}$. The evolution of the disk is followed for about $10^{4}$ years.

Using three-dimensional radiative transfer calculations of warped disk structures, Whitney et al. (2013) show that, in general, tilted thick inner disks will cast point-symmetric shadows onto the outer disk. Accordingly, our model features two point-symmetric shadows projected along the disk, with opening angles of $\delta \sim 28^{\circ}$. This value is motivated by HD 142527's case as reported by Marino et al. (2015).

The simulations were performed with the public 2D hydrodynamic code FARGO-ADSG ${ }^{4}$ (Baruteau \& Masset 2008), after implementing a non-stationary energy equation that

\footnotetext{
4 http://fargo.in2p3.fr/spip.php?rubrique9
} 
includes a blackbody radiative cooling. The code solves the Navier-Stokes, continuity, and energy equations on a staggered mesh in polar coordinates $(r, \phi)$. For detailed description of the code see Masset (2000) and Baruteau \& Masset (2008).

\subsection{Code Units and Initial Setup}

The simulations were run in code units, assuming the central star mass, $M_{0}=M_{\star}$, and $r_{0}=1 \mathrm{au}$, as the units of mass and length. The code unit of time, $t_{0}$, corresponds to the orbital period at $r_{0}$ divided by $2 \pi$, that is $t_{0}=\left(G M_{\star} / r_{0}^{3}\right)^{-1 / 2}$. The gravitational constant is $G=1$ in code units. The temperature unit is $G M_{\star} \mu m_{\mathrm{p}} /\left(k_{\mathrm{B}} r_{0}\right)$, with $\mu$ being the mean molecular weight ( $\mu=2.35$ in all our simulations), $m_{\mathrm{p}}$ as the proton mass, and $k_{\mathrm{B}}$ as the Boltzmann constant. We adopt two values for the disk-to-star mass ratio, $q=0.25$ and 0.05 .

The computational domain in physical units extends from $r=10-150$ au over $n_{r}=400$ logarithmically spaced radial cells. The grid samples $2 \pi$ in azimuth with $n_{\phi}=800$ equally spaced sectors. Gas material is allowed to outflow at the disk edges.

The initial density profile scales with $r^{-1}$ :

$$
\Sigma(r)=\Sigma_{0}\left(\frac{r_{0}}{r}\right),
$$

where the value of $\Sigma_{0}$ is set by fixing the disk mass to 0.05 and $0.25 M_{\star}\left(M_{\mathrm{disk}}=\int_{r_{\min }}^{r_{\max }} 2 \pi \Sigma(r) r d r\right)$. The disk initial aspect ratio, $H / r$, is fixed to 0.05 . All models include self-gravity. Our choice of parameters is similar to other numerical models of transition disks (e.g., Dipierro et al. 2015).

\subsection{Implementation of Shadows}

The stellar irradiation heating per unit area is given by (Fröhlich 2003):

$$
Q_{\star}^{+}=(1-\beta) \frac{L_{\star}}{4 \pi r^{2}} \cos \phi,
$$

where $\beta=0.1$ is a reflection factor (albedo) and $\phi$ is the angle formed between the incident radiation and the normal to the surface, given by $\cos \phi \simeq \frac{d H}{d r}-\frac{H}{r}$. The disk scale height is assumed to be in hydrostatic equilibrium, i.e., $H=r c_{\mathrm{s}}(T) / v_{\mathrm{k}}$, with $c_{\mathrm{s}}(T)$ as the sound speed and $v_{\mathrm{k}}$ as the Keplerian velocity. $L_{\star}$ is the stellar luminosity.

In our simulations, shadows are cast by an inner region (inside the computational domain), which blocks a fraction of the stellar irradiation $Q_{\star}^{+}$within an angle $\delta$. The irradiation heating per unit area, including shadows projected onto the disk, $Q_{d}^{+}(r, \phi)$, reads;

$$
Q_{d}^{+}(r, \phi)=\left\{\begin{array}{l}
F(t) f(\phi) Q_{\star}^{+} \\
\quad \text { if }|\phi|>(\pi-\delta / 2) \wedge|\phi|<\delta / 2 \\
Q_{\star}^{+} \text {otherwise, }
\end{array}\right.
$$

where illuminated regions are connected by a smooth azimuthal function $f(\phi)=1-A \exp \left(-\phi^{4} / \sigma^{2}\right)-A \exp \left(-(\phi-\pi)^{4} / \sigma^{2}\right)$, with $A=0.999$ and $\sigma=\delta / 10$. The time-dependent function $F(t)$ is a shadow-tapering factor which gradually enables the

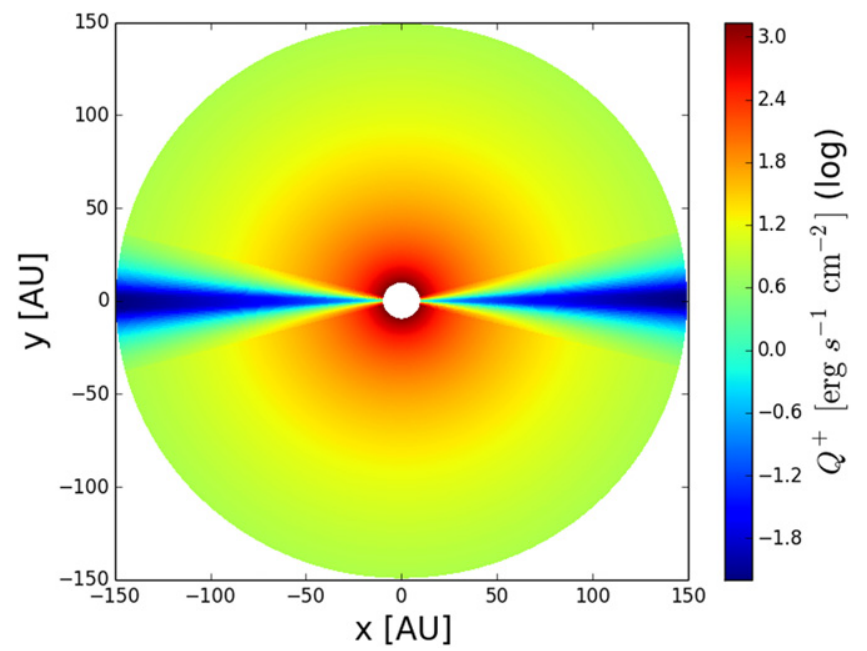

Figure 1. Initial profile for the stellar heating rate per unit area $Q_{d}^{+}(r, \phi)$ for a model with $L_{\star}=1 L_{\odot}$. The shadows subtend $0.5 \mathrm{rad}$ or $28^{\circ} .6$. The plot is in $\log$ scale with units erg $\mathrm{s}^{-1} \mathrm{~cm}^{-2}$.

shadows over a timescale of 30 orbits. $^{5}$ Figure 1 shows the stellar irradiation prescription described by Equation (3).

\subsection{The Energy Equation}

The equation for the thermal energy density, $e$, reads as (e.g., D'Angelo et al. 2003)

$$
\frac{\partial e}{\partial t}+\boldsymbol{\nabla} \cdot(e v)=-P \boldsymbol{\nabla} \cdot \boldsymbol{v}+Q_{d}^{+}-Q^{-},
$$

where $\boldsymbol{v}$ is the gas velocity, $P$ is the pressure, and $Q_{d}^{+}$is the stellar heating rate per unit area described by Equation (3). We implemented a radiative cooling per unit area function, $Q^{-}=2 \sigma T^{4} / \tau$, where $\sigma$ is the Stefan-Boltzmann constant and $\tau$ is the optical depth given by $\tau=\frac{1}{2} \Sigma \kappa$, a constant opacity given by an effective $\kappa=130 \mathrm{~cm}^{2} \mathrm{~g}^{-1}$, obtained by taking into account an average opacity for a mix of dust species, informed by spectral energy distribution fitting of HD 142527 (Casassus et al. 2015). Dependency on opacity is further discussed in Section 3. For more details about this energy prescription, see Montesinos et al. (2015).

To close the system of equations, an ideal equation of state is used:

$$
P=\Sigma T \bar{R}
$$

where $T$ is the mid-plane gas temperature and $\bar{R}=k_{\mathrm{B}} / \mu m_{\mathrm{p}}$ is the gas constant. The thermal energy density is related to the temperature through

$$
e=\Sigma T\left(\frac{\bar{R}}{\gamma-1}\right)
$$

where the adiabatic index is fixed to the diatomic gas value $\gamma=1.4$.

\footnotetext{
From the code units used, one orbit corresponds to one year.
} 

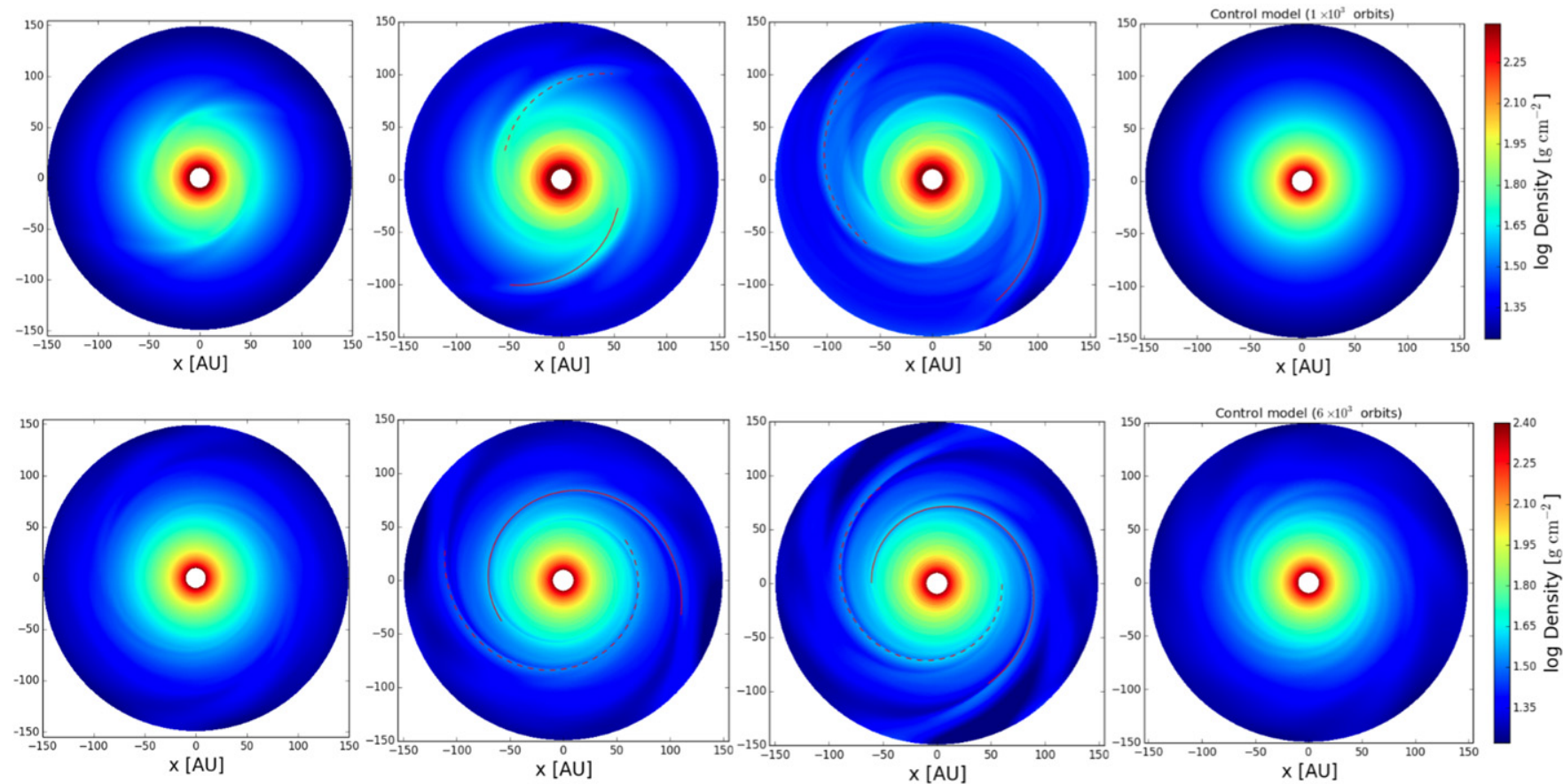

Figure 2. Density field evolution of a model disk with $M_{\mathrm{d}}=0.25 M_{\star}$. Top row: $L_{\star}=100 L_{\odot}$ model. From left to right; $150,250,500$ orbits, respectively. Bottom row: $L_{\star}=1 L_{\odot}$ model (same disk mass). From left to right; 2500, 3500, and 4000 orbits. The upper rightmost panel is a control simulation (i.e., an unshadowed model) with $L_{\star}=100 L_{\odot}$ after 1000 orbital periods. In this case, no azimuthal structures appear during the disk's evolution. The bottom rightmost panel corresponds to a control run with $L_{\star}=1 L_{\odot}$ in which the first structures appear to be caused by gravitational instabilities after $6000 t_{0}$.

\section{RESULTS}

\subsection{Spiral Structures in the Density Field}

Control simulations without shadows were performed in order to test for azimuthal structures unrelated to illumination effects. Additionally, random noise at the $\sim 0.1 \%$ level was injected into the initial surface density of these simulations in order to test for stability and fragmentation.

Azimuthal features due solely to the disk self-gravity develop in control runs with $M_{\mathrm{d}}=0.25 M_{\star}$ and $L_{\star}=1 L_{\odot}$, on timescales of $\gtrsim 10^{3}$ orbits. This is expected for such gravitationally unstable disks (Dipierro et al. 2015). For control models with $M_{\mathrm{d}}=0.05 M_{\star}$ (gravitationally stable), no spiral structures emerge, independent of the strength of the stellar irradiation field.

When shadows are enabled, spiral-like structures emerge in the surface density of a simulated $0.05 M_{\star}$ disk irradiated by a $100 L_{\odot}$ star. The onset of these structures occurs approximately after 130 orbital periods. On the other hand, no spirals appear if the $0.05 M_{\star}$ disk is illuminated by only $1 L_{\odot}$.

We consider the onset of the shadow-induced spirals to be the time at which their scale heights suffice $\delta H / H \simeq 0.3$. This criterion is evaluated within the first 10-20 au of radius from the star. The perturbation on the scale height is calculated as $\delta H \equiv H-H_{0}$, where $H_{0}$ represents the background scale height without shadows. We note that a relative change of $\sim 0.2$ in scale height is sufficient to produce detectable azimuthal signatures in scattered light predictions (Dong et al. 2015b; Juhász et al. 2015).

For the more massive $0.25 M_{\star}$ disk, with $L_{\star}=1 L_{\odot}$, the first non-axisymetric structures appear approximately after 2500 orbits. For the same disk mass, but increasing stellar irradiation to $100 L_{\odot}$, the first spirals arise shortly after only 150 orbits.
These different timescales will be discussed in the next subsection.

Figure 2 shows the impact of different stellar irradiation values on the evolution of the density field, for a $0.25 M_{\star}$ disk. The top row shows the $100 L_{\odot}$ model after 150,250 , and 400 orbits (from left to right, respectively), while the bottom panels show the $1 L_{\odot}$ model at 2500, 3500, and 4000 orbits (from left to right). Each first snapshot corresponds to the moment spirals appear for the first time, as defined above. The rightmost top and bottom panels correspond to control runs, in which no shadows are present.

In order to test the dependency of the disk evolution with the opacity, we also explore the case with $\kappa=1 \mathrm{~cm}^{2} \mathrm{~g}^{-1}$, finding the same results. This insensibility to the opacity, in order to produce shadow-induced spiral arms, is not surprising. The radiative cooling is computed according to $T^{4} / \tau$, therefore changes in $\kappa$ imply (roughly speaking) changes in the temperature field of about $\sim \kappa^{1 / 4}$. Moreover, the amplitude of a thermal perturbation responsible for spiral development is given by $\delta T / T$ (see the next section); therefore, in an equilibrium situation in which $Q^{+} \sim Q^{-}$, the assumption of a constant opacity results in a thermal amplitude perturbation independent of the opacity choice.

Our 2D simulations are not radiation hydrodynamics but just an ideal approximation. The role of the opacity will be considered in a future work, where we will study the illumination-induced spirals using full 3D radiation hydrodynamic simulations.

\subsubsection{Pitch Angle}

The spiral arms' pitch angles were computed by fitting the spirals with an Archimedean equation $r(\phi)=A_{0}+A_{1} \phi^{n}$. The pitch angle $\Pi$ is then obtained through $\tan \Pi=(1 / r) d r / d \phi$. 
Table 1

Main Spiral and Disk Characteristics

\begin{tabular}{|c|c|c|c|c|}
\hline Model Parameters & Orbit Number & $\begin{array}{l}\text { Spiral Parameters } \\
A_{0}, A_{1}, n, \Pi^{\circ}\end{array}$ & $\begin{array}{c}\text { Toomre }^{\mathrm{a}} \\
Q_{\min }\end{array}$ & $\begin{array}{l}\text { Birth Timescale of } \\
\text { the Structures }{ }^{\mathrm{b}} \text { (in Orbits) }\end{array}$ \\
\hline$M_{\mathrm{d}}=0.05 M_{\star} ; L_{\star}=1 L_{\odot}$ & $\begin{array}{l}3500 \\
4000\end{array}$ & $\begin{array}{l}\mathrm{n} / \mathrm{a} \\
\mathrm{n} / \mathrm{a}\end{array}$ & $\begin{array}{l}1.1 \\
1.1\end{array}$ & $\mathrm{n} / \mathrm{a}$ \\
\hline$M_{\mathrm{d}}=0.05 M_{\star} ; L_{\star}=100 L_{\odot}$ & $\begin{array}{l}250 \\
500\end{array}$ & $\begin{array}{l}68.7 ; 14.6 ; 2.1 ; 22.59 \pm 3.5 \\
56.9 ; 4.2 ; 1.64 ; 13.82 \pm 3.5\end{array}$ & $\begin{array}{l}3.4 \\
2.6\end{array}$ & $\sim 150$ \\
\hline$M_{\mathrm{d}}=0.25 M_{\star} ; L_{\star}=100 L_{\odot}$ & $\begin{array}{l}250 \\
500\end{array}$ & $\begin{array}{l}60.3 ; 28.2 ; 1.35 ; 15.04 \pm 2.5 \\
87.2 ; 17.8 ; 1.43 ; 13.09 \pm 2.0\end{array}$ & $\begin{array}{l}1.0 \\
1.2\end{array}$ & $\sim 150$ \\
\hline
\end{tabular}

Notes. The spirals can be described with the Archimedean function $r(\phi)=A_{0}+A_{1} \phi^{n}$, where the pitch angle is given by $\tan \Pi=(1 / r) d r / d \phi$. The value $A_{0}$ (in au) gives the inner radius of the spirals, which, in our models extends to the outskirts of the disk $(\sim 150 \mathrm{au})$.

${ }^{a}$ Minimum local value of the Toomre parameter at the given orbit number.

${ }^{\mathrm{b}}$ Orbit number when spirals are distinguishable for the first time in the density field (see Section 3.1).
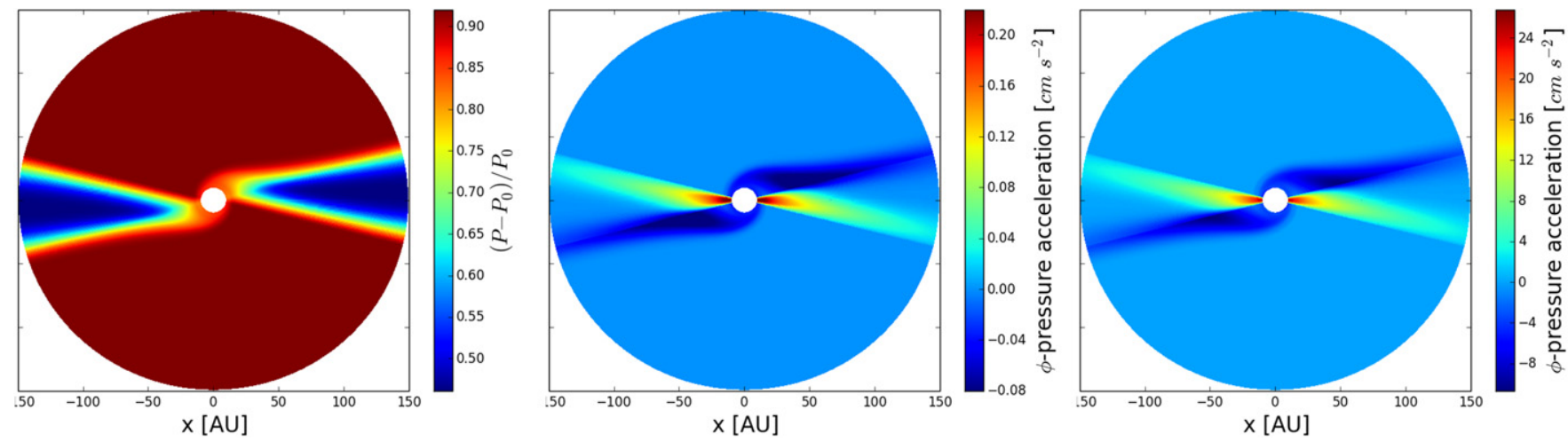

Figure 3. Left panel: illustration of how the illumination effect impacts the pressure field (i.e., $\left(P-P_{0}\right) / P_{0}$, where $P_{0}$ is the background pressure field without the shadows) after 30 orbits for a model disk with $L_{\star}=100 L_{\odot}$ and $0.25 M_{\star}$. Pressure decreases at the shadows' positions. Middle and right panels: azimuthal pressure acceleration $\left(a_{\phi}=-(1 / r \Sigma) \partial P / \partial \phi\right)$ after 30 orbits for models with $L_{\star}=1 L_{\odot}$ and $L_{\star}=100 L_{\odot}$, respectively (same disk mass for both models).

From this parameterized curve, a global pitch angle is calculated as the mean value along the curve.

$\Pi$ varies over time for different model parameters. The shadow-induced spiral arms extend from $A_{0}(\sim 60-90 \mathrm{au}$, see Table 1) to the outer region of the disk $\sim 150$ au (see Figure 2), as opposed to spirals resulting from GI, which persist over scales $\lesssim 100$ au (e.g., Dong et al. 2015b). Table 1 summarizes the most important features of the shadow-induced spirals.

\subsection{Formation of Spiral-like Structures from Shadows}

Perturbations in a differentially rotating gaseous disk tend to wind up into spiral patterns (e.g., see the control model in the bottom rightmost panel in Figure 2). In our models, projected shadows act as forcing perturbations, which, efficiently and independently of the presence of random sources of symmetry breaking (e.g., gravitational instabilities), produce spirals. Because of the point-symmetric nature of this perturbation, density waves are excited with $m=2$ morphology. Each shadow appears to trigger one spiral arm.

Shortly after the shadows have been cast onto the disk, and before the disk thermalizes, the gas pressure (Equation (5)) plummets at the shadowed regions. Figure 3 (left panel) shows the relative change in pressure $\left(P-P_{0}\right) / P_{0}$, where $P_{0}$ is the background unshadowed gas pressure. This snapshot corresponds to a $100 L_{\odot}$ and $0.25 M_{\star}$ model disk immediately after shadows are enabled. Figure 3 shows the azimuthal pressure acceleration, $\boldsymbol{a}_{\phi}$, after 30 orbits for two $0.25 M_{\star}$ disk models with $L_{\star}=1 L_{\odot}$ and $100 L_{\odot}$, middle and right panels, respectively. The pressure acceleration magnitude increases with stellar irradiation: the maximum value for the pressure acceleration increases from 0.2 to $24 \mathrm{~cm} \mathrm{~s}^{-2}$ when the stellar irradiation passes from $L_{\star}=1 L_{\odot}$ (middle) to $L_{\star}=100 L_{\odot}$ (right panel).

As the gas in the disk flows around the star (in a counterclockwise direction for all figures), it periodically enters and exits shadowed regions. At the interfaces between shadowed and fully illuminated sections, the gas is subjected to an azimuthal acceleration due to pressure gradients (i.e., $\left.\boldsymbol{a}_{\phi}=-(1 / r \Sigma(r)) \nabla_{\phi} P\right)$. As the gas enters a shadowed region (coming from high to low pressure), it experiences a positive azimuthal acceleration (anticlockwise). On the other hand, as it exits the shadow (moving from low to high pressure), the gas experiences a negative azimuthal acceleration (clockwise). The difference between the gain and loss of azimuthal acceleration is uneven, resulting in a net azimuthal acceleration in the counterclockwise direction (gas rotation direction), which varies with radius within the first $\sim 50 \mathrm{au}$. This creates a strong source of axisymmetry breaking, locally pushing the gas to move faster around the central star at the inner regions of the disk, piling up material at the shadow's frontier close to this 
inner region, which then leads to the formation of a trailing mode of spiral-like structures outspread by differential rotation.

Gas pressure acceleration depends on stellar luminosity as more luminous stars feed more energy into their disks, raising the temperature of the illuminated sections while leaving the shadowed region unaffected. This produces higher contrasts in the pressure field. This is the reason why spirals appear early ( $\sim 130$ orbits) in models with $L_{\star}=100 L_{\odot}$, when compared to $1 L_{\odot}$ models in which the first spirals emerge only after $\sim 2500$ orbits. The key factor is the contrast in pressure between dark and illuminated regions.

Depending on the gas cooling time, thermal perturbations can wear off over time. As the disk thermalizes, the illumination pattern becomes less effective at forcing a perturbation on the gas. In our simulations, this tendency to homogenize is seen after $10^{5}$ orbits. Ultimately, the induced spiral arms tend to blend and lose contrast with the background gas unless GI kick in. In these large viscous timescales, GIinduced spiral structures are expected to remain quasi-steady (e.g., Dipierro et al. 2015).

\subsection{Gravitational Instabilities}

GI can be locally characterized by the Toomre parameter ${ }^{6}$ given by $Q=\frac{c_{\mathrm{s}} \Omega}{\pi G \Sigma}$, where $c_{\mathrm{s}}, \Omega$, and $\Sigma$ correspond to sound speed, angular velocity, and surface density, respectively (Toomre 1964). The disk becomes unstable when $Q<Q_{\text {crit }}$, where $Q_{\text {crit }}$ defines the range for which a disk is marginally unstable: $1 \lesssim Q_{\text {crit }} \lesssim 2$.

According to the local Toomre parameter, denser and colder regions of the disk tend to have lower $Q$ values. The $M_{\mathrm{d}}=0.05 M_{\star}$ and $L_{\star}=100 L_{\odot}$ model is stable everywhere in the disk, with a minimum $Q$ value of 3.35 . The most unstable model $\left(M_{\mathrm{d}}=0.25 M_{\star}\right.$ and $L_{\star}=1 L_{\odot}$ attains a minimum local value of $Q=0.5$. It is important to note that spiral structures emerge even if we disable self-gravity from our simulations. The shadow-induced spirals do not require the presence of GI for their development.

\subsection{Radiative Transfer}

We input our simulations into a $3 \mathrm{D}$ radiative transfer code to produce scattered light predictions in the $H$-band $(1.6 \mu \mathrm{m})$. We used the RADMC $3 \mathrm{D}^{7}$ code (version 0.39), assuming that the dust follows the same density field as the gas in the simulation, with a gas-to-dust ratio of 100 . The dust distribution model consists of a mix of two common species: amorphous carbon and astronomical silicates. We used the Mie model (homogeneous spheres) to compute dust opacities for anisotropic scattering (Bohren \& Huffman 1983). The optical constants for amorphous carbon (intrinsic grain densities $2 \mathrm{~g} \mathrm{~cm}^{-3}$ ) were taken from Li \& Greenberg (1997), and those for silicates (intrinsic grain densities $4 \mathrm{~g} \mathrm{~cm}^{-3}$ ) were taken from Draine \& Lee (1984).

To produce a three-dimensional (3D) volume, the vertical density structure was solved assuming hydrostatic equilibrium. The disk scale height is obtained from the temperature field (Section 2.2).

\footnotetext{
6 For extremely massive disks, $\Omega$ should be replaced by the epicyclic frequency $\kappa$, defined by $\kappa^{2}=\left(1 / r^{3}\right) d / d r\left(r^{4} \Omega^{2}\right)$. In our case, $\kappa \approx \Omega$.

7 http://www.ita.uni-heidelberg.de/ dullemond/software/radmc-3d/
}

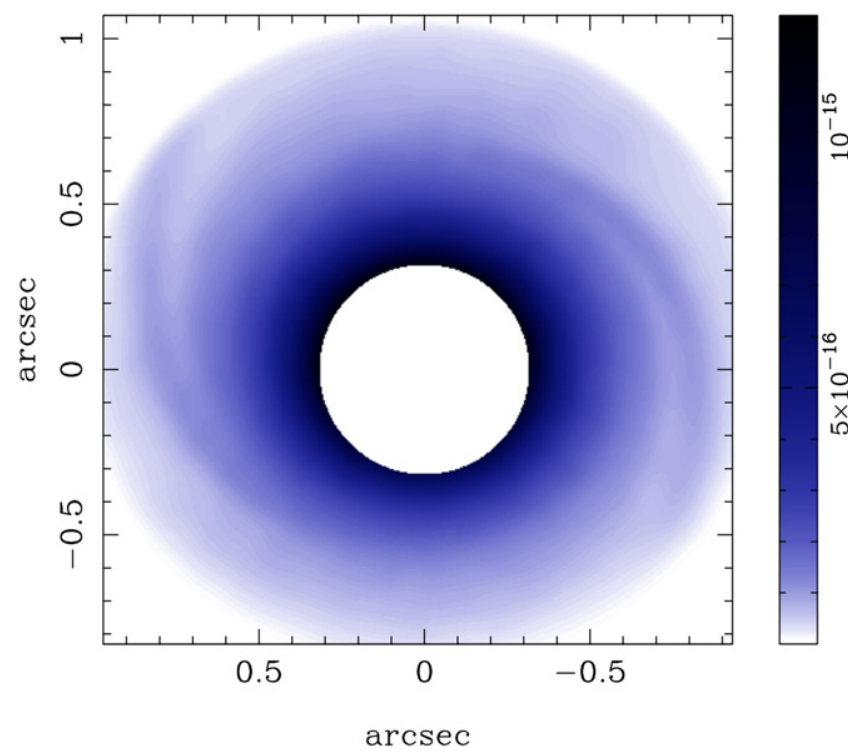

Figure 4. Scattered light prediction for the $H$-band $(1.6 \mu \mathrm{m})$ obtained from $3 \mathrm{D}$ radiative transfer calculations. The hydrodynamical input corresponds to a model with a disk mass of $0.25 M_{\star}$, and $1 L_{\odot}$ star. Color stretch is linear, with units of $\mathrm{erg} \mathrm{cm}^{-2} \mathrm{~s}^{-1} \mathrm{~Hz}^{-1} \mathrm{ster}^{-1}$.

The temperature in the vertical direction is assumed to be constant and equal to the mid-plane temperature with $T_{\text {gas }}=T_{\text {dust }}$. The final image prediction is rendered using a second order volume ray-tracing.

Figure 4 shows a model prediction in the $H$-band based on a $0.25 M_{\star}$ disk with stellar irradiation of $1 L_{\odot}$, after it had evolved for 3500 orbits. We recover the spiral arms in scattered light. We also obtain observable spiral structures in the $L$-band. The scattered light spirals follow the same equation as the one obtained from the density field, i.e., $\Pi=13^{\circ} .82$ (see Figure 2, bottom row, orbit 3500 , and/or Table 1 ).

It is worth mentioning that the assumption of hydrostatic equilibrium in 2D models likely underestimates the contrast of spirals images when compared with with full 3D hydrodynamical models (Zhu et al. 2015). In that case, the illumination effects impacting the disk should produce even more prominent scattered light images of spirals than those reported here.

\section{CONCLUDING REMARKS}

We investigated the hydrodynamical consequences of illumination effects in transitional disks, such as shadows cast onto a circumstellar disk, focusing on the development of spiral structures. The evolution of a passive disk was calculated using 2D hydro simulations, including stellar irradiation values of 1 and $100 L_{\odot}$, and with two point-symmetric shadows cast within an opening angle of $28^{\circ}$ for disk masses of $M_{\mathrm{d}}=0.05$ and $M_{\mathrm{d}}=0.25 M_{\star}$. Pressure gradients due to temperature differences between obscured and illuminated regions induce spiral structures in the density field. These spirals emerge independent of whether self-gravity is enabled or not. The structures observed in the density field can be characterized by an Archimedean spiral that attains nearly constant pitch angles of $\sim 11^{\circ}-14^{\circ}$, and extends from $\sim 60$ au to the outer disk rim. Radiative transfer calculations, for a model with $0.25 M_{\star}$ disk mass and stellar irradiation of $1 L_{\odot}$, predict that these shadowinduced spirals should be detectable in $H$-band scattered light images. 
Recently, Rafikov (2016) presented an analytical study of the effect of density waves on protoplanetary disks. They found that density waves with a contrast of order unity, such as the ones resulting from our models, produce an enhancement in the accretion rate that could explain the large cavities observed in some transition disks. In HD 142527, the inner disk casts a shadow that explains the observed dips in the outer disk (Marino et al. 2015), and, as shown in this letter, drives spiral waves (Casassus et al. 2012a; Christiaens et al. 2014). Following Rafikov (2016), the enhanced accretion produced by these spirals could have depleted the inner $\sim 100$ au of this disk. The HD 100453 disk (Wagner et al. 2015), which we suggest bears strong similarities to HD 142527, as spirals that are seen stemming away from dark regions in the outer disk, might also fit this scenario. A more detailed study of the interplay between the different processes will be investigated in a follow-up paper.

We thank Clément Baruteau for very useful comments on this paper. Financial support was provided by Millennium Nucleus grant RC130007 (Chilean Ministry of Economy). M. M. acknowledges support from CONICYT-Gemini grant 32130007. S.C., S.P., and J.C. acknowledge financial support provided by FONDECYT grants 1130949, 3140601, and 1141175. The authors also thank the referee suggestions that have improved this letter.

\section{REFERENCES}

Baruteau, C., \& Masset, F. 2008, ApJ, 678, 483

Benisty, M., Juhasz, A., Boccaletti, A., et al. 2015, A\&A, 578, L6

Boccaletti, A., Pantin, E., Lagrange, A.-M., et al. 2013, A\&A, 560, A20
Bohren, C. F., \& Huffman, D. R. 1983, Absorption and Scattering of Light by Small Particles (New York: Wiley)

Casassus, S., Marino, S., Pérez, S., et al. 2015, ApJ, 811, 92

Casassus, S., Perez, M. S., Jordán, A., et al. 2012a, ApJL, 754, L31

Casassus, S., Wright, C. M., Marino, S., et al. 2015, ApJ, 812, 126

Christiaens, V., Casassus, S., Perez, S., van der Plas, G., \& Ménard, F. 2014, ApJL, 785, L12

Clampin, M., Krist, J. E., Ardila, D. R., et al. 2003, AJ, 126, 385

D’Angelo, G., Henning, T., \& Kley, W. 2003, ApJ, 599, 548

Dipierro, G., Pinilla, P., Lodato, G., \& Testi, L. 2015, MNRAS, 451, 974

Dong, R., Hall, C., Rice, K., \& Chiang, E. 2015a, ApJL, 812, L32

Dong, R., Zhu, Z., Rafikov, R. R., \& Stone, J. M. 2015b, ApJL, 809, L5

Draine, B. T., \& Lee, H. M. 1984, ApJ, 285, 89

Espaillat, C., Ingleby, L., Hernández, J., et al. 2012, ApJ, 747, 103

Fröhlich, H.-E. 2003, S\&W, 42, 98

Fukagawa, M., Hayashi, M., Tamura, M., et al. 2004, ApJL, 605, L53

Fukagawa, M., Tamura, M., Itoh, Y., et al. 2006, ApJL, 636, L153

Garufi, A., Quanz, S. P., Avenhaus, H., et al. 2013, A\&A, 560, A105

Grady, C. A., Muto, T., Hashimoto, J., et al. 2013, ApJ, 762, 48

Grady, C. A., Polomski, E. F., Henning, T., et al. 2001, AJ, 122, 3396

Grady, C. A., Woodgate, B., Bruhweiler, F. C., et al. 1999, ApJL, 523, L151

Juhász, A., Benisty, M., Pohl, A., et al. 2015, MNRAS, 451, 1147

Li, A., \& Greenberg, J. M. 1997, A\&A, 323, 566

Lodato, G., \& Rice, W. K. M. 2004, MNRAS, 351, 630

Marino, S., Perez, S., \& Casassus, S. 2015, ApJL, 798, L44

Masset, F. 2000, A\&AS, 141, 165

Montesinos, M., Cuadra, J., Perez, S., Baruteau, C., \& Casassus, S. 2015, ApJ, 806,253

Muto, T., Grady, C. A., Hashimoto, J., et al. 2012a, ApJL, 748, L22

Muto, T., Grady, C. A., Hashimoto, J., et al. 2012b, ApJL, 748, L22

Pohl, A., Pinilla, P., Benisty, M., et al. 2015, MNRAS, 453, 1768

Rafikov, R. R. 2016, arXiv:1601.03009

Toomre, A. 1964, ApJ, 139, 1217

Wagner, K., Apai, D., Kasper, M., \& Robberto, M. 2015, ApJL, 813, L2

Wahhaj, Z., Cieza, L. A., Mawet, D., et al. 2015, A\&A, 581, A24

Whitney, B. A., Robitaille, T. P., Bjorkman, J. E., et al. 2013, ApJS, 207, 30

Zhu, Z., Dong, R., Stone, J. M., \& Rafikov, R. R. 2015, ApJ, 813, 88 